Proceedings

\title{
Electrochemical Impedance Spectroscopy Characterization of Label-Free Biosensors for the Detection of HER2 in Saliva ${ }^{\dagger}$
}

\author{
Imad Abrao Nemeir 1.2,*, Lynn Mouawad 1, Joseph Saab 1, Walid Hleihel 1, Abdelhamid Errachid ${ }^{2}$ \\ and Nadia Zine ${ }^{2, *}$ \\ 1 Faculty of Sciences, Holy Spirit University of Kaslik, Jounieh 446, Lebanon; \\ 2 Institut des Sciences Analytiques, Université de Lyon, Claude Bernard Lyon 1, UMR 5280, CNRS-5, \\ rue de la Doua, 69100 Villeurbanne, France; \\ * Correspondence: Imad-nmeir@hotmail.com (I.A.M.); nadia.zine@univ-lyon1.fr (N.Z.) \\ + Presented at the 1st International Electronic Conference on Biosensors, 2-17 November 2020; Available \\ online: https://iecb2020.sciforum.net/.
}

Received: date; Accepted: date; Published: date

\begin{abstract}
Breast cancer is the leading cancer site for women with 2 million new yearly infections and more than half a million dead worldwide. Human Epidermal Receptor 2 (HER2) is a prominent breast cancer biomarker that indicates aggressive cancer and is often associated with a bad prognosis and low survival rates. However, current detection methods for HER2 are often timeconsuming, expensive, and require a high level of expertise. Biosensors are devices that turn biological interaction into a readable electronic signal; they are known for their high specificity and selectivity for low concentration, as well as their low cost and ease of use, thus making them a better alternative to traditional methods. Also, Saliva is becoming a better alternative for blood for the detection of biomarkers due to its non-invasive collection in large quantities with simple collection methods with a richness in disease biomarkers including HER2. Thus this project aims to develop a label-free, low cost, electrochemical biosensor for the detection of HER2 in saliva. This was done by first depositing diazonium salt onto a screen-printed electrode (SPE) through cyclic voltammetry, then immobilizing anti-HER2 antibodies on the activated SPE using the EDC/NHS protocol [1]. HER2 biomarker concentrations were detected using electrochemical impedance spectroscopy inside a microfluidic system. The biosensor showed a higher linear detection of HER2 ( $Y=0.0062 \times$ $+0.1066 / \mathrm{R}^{2}=0.9909$ ) in its physiological concentration range of 5 and $40 \mathrm{pg} / \mathrm{mL}$ when compared to other interference proteins: Epidermal Growth Factor Receptor $\left(Y=0.0016 x+0.0188 / R^{2}=0.8072\right)$ and Human Epidermal Receptor $3\left(Y=\left(0.0035 x+0.0225 / R^{2}=0.1302\right)\right.$. The biosensor was then used to detect $10 \mathrm{pg} / \mathrm{mL}$ of HER2 concentration in real saliva using the standard addition methods $(\mathrm{Y}=$ $\left.0.0118 x+0.1282 / R^{2}=0.9876\right)$.
\end{abstract}

\section{Introduction}

With 2 million new cases in 2018, Breast cancer is the leading type of cancer for women worldwide [1-3].

Human epithelial receptor 2 (HER2, ErbB2) is a receptor protein typically found on the surface of the cells, it consists of three functional domains: the extracellular domain (ECD) can bind with other members of HER family; a lipophilic transmembrane domain, and the intracellular domain containing tyrosine kinase activity. HER2 is amplified in nearly $20 \%$ of breast cancer patients and it is an indicator for a worse prognosis. Regardless, anti-HER2 therapy (trastuzumab or lapatinib) is administered to improve patient's survival. HER2 concentrations is determined using immunohistochemistry (IHC) or fluorescent in situ hybridization (FISH) on tumor tissue following 
biopsy. However, this detection method is fraught with problems, first, due to biopsy's complex nature, false negative or positive results are an issue with these techniques, and, biopsy is extremely invasive technique that carries a lot of risks [4-7].

A solution to the above-mentioned problem is the use a different biological medium for the detection of HER2; this is possible due to the detachability of the extracellular part into the extracellular matrix due to proteolytic cleavage that occur on the cell's surface [8], and while blood is usually the favored medium for the detection of biomarkers, saliva is seen as a great alternative [912]; saliva has several advantages on other biomarker rich biological mediums due to its availability in large quantities, its richness in biomarkers and its ease of collection without needles [13,14]. A few of the hurdles facing salivary detection have unfortunately hindered their use for clinical detection of a large number of diseases; from the low concentration of HER2 within the fM range [15-17], to issues and difficulties with the salivary matrix [18]. But above all, the lack of a technique for the low cost HER2 detection that is optimized for the detection in the salivary matrix [11].

Biosensors are devices whose function is to turn biological signals into a physical one; biosensors have shown to exhibit high specificity and selectivity toward their target biomarker in low concentrations while remaining cost effective and easy to use, making them a better alternative to traditional techniques for biomarker detection [11,19].

Of all the techniques used in biosensors for the recognition of biomarkers, Electrochemical impedance spectroscopy (EIS) seems to be one of its most prominent. Its ability to investigate both bulk and interfacial electrical properties of three electrode systems have been used to determine quantitative biorecognition in different biosensors [20,21]. The mechanism of which EIS is used in biosensor detection is rather simple in principle: if biorecognition of the biomarker occurs at the adapted electrode's surface, the electrochemical interfacial properties of said electrode changes thus providing a fingerprint of the above mentioned change, creating a detection signal [22-24].

One of the techniques used to create the above mentioned electrodes used for EIS detection involves deposition layer-by-layer of a paste or an ink containing electrochemically conductive materials (gold, silver, carbon...) on a solid surface (ceramic, plastic...) through a screen or a mesh with a well-defined geometry. Electrodes made from this technique are known as Screen-Printed electrode (SPE) and they offers a promising approach toward creating easy to use electrochemical biosensors [25].

In this paper we present the development of an electrochemical biosensor for the detection of HER2 in human saliva using a biofunctionalized gold integrated SPE; The SPE was functionalized using Cyclic voltammetry (CV) and EIS was used in HER2 detection. The biosensor managed to detect HER2 within the range of 5 to $40 \mathrm{pg} / \mathrm{mL}$ in PBS, which is within the detection range of previous articles, all the while showing higher sensitivity toward HER2 when compared to the detection of other interferences (EGFR and HER3), and finally, HER2 was then detected in real saliva using the standard addition method.

\section{Materials and Method}

\subsection{Chemical Products}

The reagents used in this research are: 4-aminobenzoic acid (ABA), sodium nitrite $\left(\mathrm{NaNO}_{2}\right)$, hydrochloric acid $(\mathrm{HCl})$, N-hydroxysuccinimide (NHS),

$\mathrm{N}$-(3-dimethylaminopropyl)-N-ethyl-carbodiimide hydrochloride (EDC), ethanolamine, phosphate-buffered saline (PBS), and (Fe2+Fe3+) were purchased from Sigma Aldrich (Lebanon). HER2, epidermal growth factor receptor (EGFR), and Human epidermal receptor 3 (HER3) antigens and anti-HER2 antibody were purchased from R\&D System (Lebanon).

\subsection{Antibodies ( $A b)$ and Antigen $(A g)$ Solutions}

As per the supplier's protocol, the antibodies and the antigens have been diluted in PBS (pH 7.4) to acquire a stock solution with a final concentration of $10 \mu \mathrm{g} / \mathrm{mL}$. for the antigens (HER2, EGFR, 
HER3) and $0.5 \mathrm{mg} / \mathrm{mL}$ for anti-HER2 antibody (anti-HER2), which were then divided into aliquots and stored at $-20{ }^{\circ} \mathrm{C}$. The stock solutions for HER2 was then further diluted in PBS to form the standard solutions used in the analysis. The standard solutions of HER2 were prepared by diluting the appropriate amount of a stock solution $(50 \mu \mathrm{g} / \mathrm{mL})$ in PBS in order to obtain the necessary concentrations of HER, EGFR, and HER3 (5, 10, 20, 40 pg/mL).

\subsection{Screen Printed Electrodes}

The biosensor platform that was used for the detection of HER2 is a lab-on-chip made by Micrux, Spain, with eight units of integrated SPE that make up a contained electrochemical system of one gold working electrode, one silver/silver chloride reference electrode and one carbon counter electrode. These chips were previously fabricated and characterized for detection in complex mediums; an image of it can be seen in Figure 1A [26]. A microfluidic system is then used to place the chips in for ease of use as seen in Figure 1B.

(A)

(B)

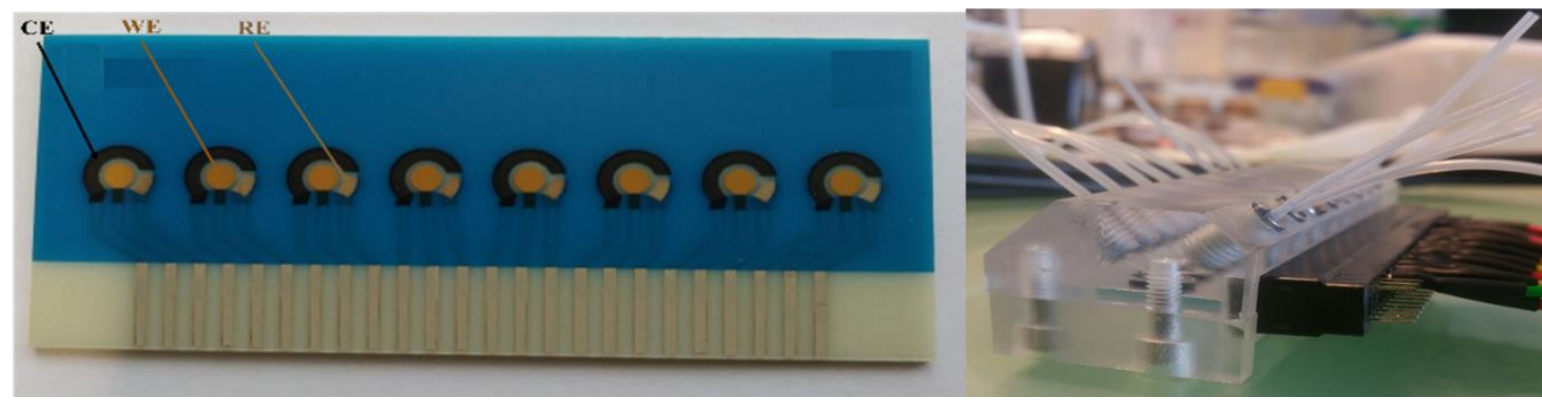

Figure 1. (A) Chip platform containing 8 integrated screen printed electrode of a silver/silver oxide reference electrode (RE) a carbon counter electrode (CE) and a gold working electrode (WE) each. (B) The microfluidic system used for the management of the experiment.

\subsection{Electrochemical Characterization}

All measurements were done at room temperature $(25 \pm 2 \circ \mathrm{C})$ in a Faraday box using a PalmSense 4 potentiostat (Palmsense B.V., Netherlands) the software used is PSTrace (version 5.7, Palmsense B.V., Netherlands).

$\mathrm{CV}$ was used to characterize the SPE using the probe $5 \mathrm{mM}$ of $\mathrm{K}_{3}\left[\mathrm{Fe}(\mathrm{CN})_{6}\right] / \mathrm{K}_{4}\left[\mathrm{Fe}(\mathrm{CN})_{6}\right]$ in PBS buffer at $\mathrm{pH}=7.4$. The scan rate was $60 \mathrm{mV} / \mathrm{s}$ with a window of -0.4 to $0.6 \mathrm{~V}$ and was repeated for 3 scans.

EIS was used to characterize the electrochemical properties of the SPE during the measurement of HER2 concentrations, this was done in $5 \mathrm{mM}$ of $\mathrm{K}_{3}\left[\mathrm{Fe}(\mathrm{CN})_{6}\right] / \mathrm{K}_{4}\left[\mathrm{Fe}(\mathrm{CN})_{6}\right]$ in PBS buffer at pH7.4. The applied potential was kept at $0.174 \mathrm{~V}$ versus the integrated reference electrode, an alternative potential of $10 \mathrm{mV}$, and a frequency window between 0.5 to $10000 \mathrm{~Hz}$. Data analysis was accomplished using the open access software EIS spectrum analyzer.

\subsection{Bio-Functionalization of the WE}

Biofunctionalization of the WE was carried out in two major steps, the first is the electrodeposition of ABA on the gold surface, the second is the immobilization of the antibody antiHER2 on the $-\mathrm{COOH}$ group of the electrodeposited ABA.

In order to observe the effects of $\mathrm{ABA}$ electrodeposition, $\mathrm{CV}$ was used in the parameters that were mentioned in part 2.4 in order to characterize the working electrode before and after deposition. Electrodeposition of ABA was carried out by first: $1 \mathrm{mM} \mathrm{ABA}$ solution was dissolved in $0.5 \mathrm{M} \mathrm{HCl}$ through 1 min of sonification, then $1 \mathrm{mM} \mathrm{NaNO}_{2}$ was added and the solution was kept on ice for 5 min to form the diazonium radicals. This is directly followed by ABA electrodeposition using CV with the following parameters: 15 scan cycles with a scan rate of $0.1 \mathrm{~V} / \mathrm{s}$ from -0.4 to $0.4[27,28]$. The resulting electrodeposition caused the blocking of the gold surface with $\mathrm{ABA}$, creating a weak 
electron kinetic transfer. This results in the disappearance of the oxydoreduction peaks of $\mathrm{Fe}^{2+} / \mathrm{Fe}^{3+}$ that are present in the solution upon characterization of the SPE using CV as seen in Figure 2.

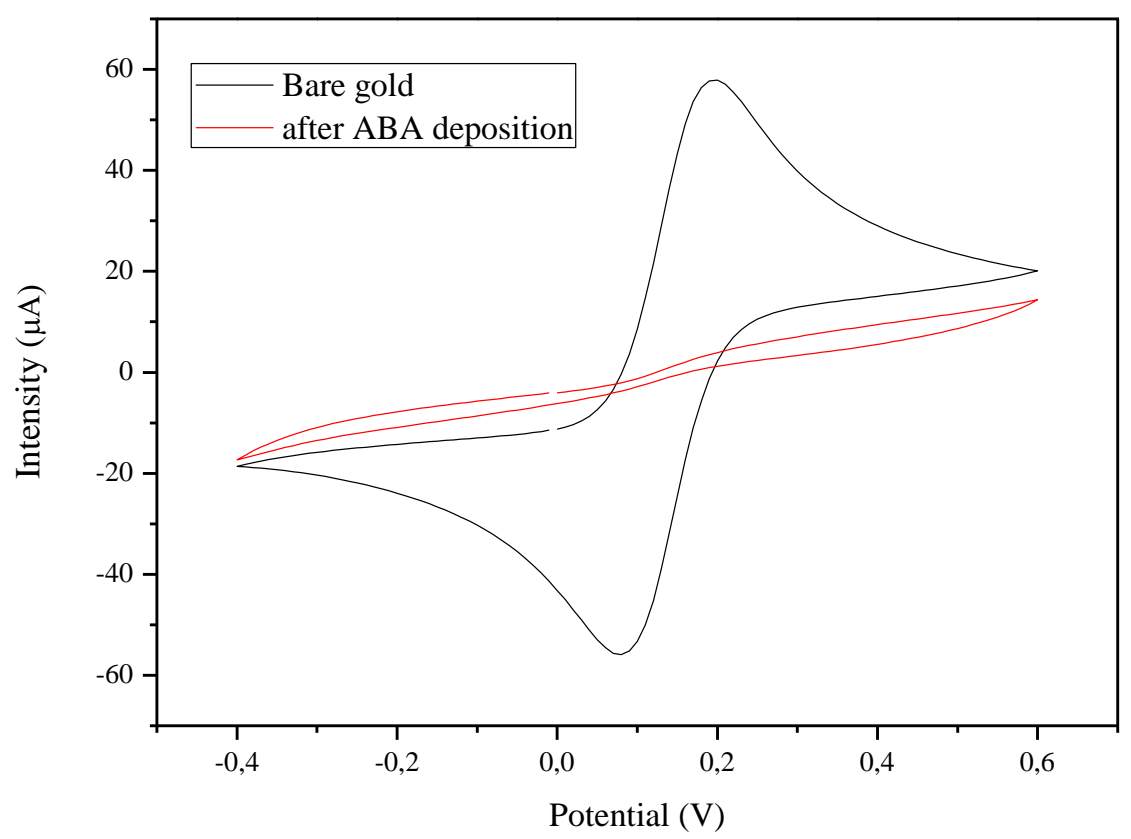

Figure 2. Effect of the electrodeposition of ABA on the electrode surface. CV of the gold WE in $5 \mathrm{mM}$ $\mathrm{K} 3[\mathrm{Fe}(\mathrm{CN}) 6] / \mathrm{K} 4[\mathrm{Fe}(\mathrm{CN}) 6]$ before (black) and after (red) electrodeposition.

Anti-HER2 was then immobilized on top of the ABA by first activating the carboxylic acid groups of ABA molecules with EDC/NHS coupling agents through incubating them (electrodes) in EDC $(0.4 \mathrm{M}) / \mathrm{NHS}(0.1 \mathrm{M})$ in water for $40 \mathrm{~min}$. Afterwards the device was washed with PBS to eliminate excess EDC/NHS, and immediately incubated with the antibody anti-HER2 in PBS (10 $\mu \mathrm{g} / \mathrm{mL}$ ) for $40 \mathrm{~min}$ at room temperature, afterwards the remaining carboxylic groups were deactivated by incubating the SPE in ethanolamine solution (1\%o in PBS) at room temperature for 30 $\min [29]$.

\subsection{HER2 Detection in Real Saliva}

To perform the detection in real saliva, $1 \mu \mathrm{g} / \mathrm{mL}$ HER2 stock solution was dissolved in $1 \mathrm{~mL}$ of a real saliva; the real saliva was taken from a healthy male subject aged 28 years at 10 am after breakfast. This created a solution of real saliva with an HER2 concentration of $1 \mathrm{ng} / \mathrm{mL}$ HER2. This step is followed by dissolving the above-mentioned saliva solution in equal volume in 4 different aliquots, then diluting in each aliquot different volumes of the stock HER2 solution of $10 \mu \mathrm{g} / \mathrm{mL}$ in each aliquot to create the range of concentration $(0,5,10,20$, and $40 \mathrm{pg} / \mathrm{mL}$ of added standard HER2 concentration). 


\section{Results}

\subsection{Detection and Interferences of HER2 in PBS}

Detection of HER2 concentrations are presented in Bode plot format in Figure 3A. Bode plot allows the presentation of both the total impedance described as $\mathrm{Z}(\mathrm{Ohm})$ and the phase angle (degree) shifts in relations to base 10 logarithms of the frequencies (log freq) of a given spectrum. The equivalent circuit used for the detection can be found in Figure 3A inlet.

Fitting was done using EIS analyzer, an open source program used for the deduction of the value of each component of a given equivalent circuit. The biosensor is being designed to use the resistive current, as such the area with the greater significant differences in the resistance between each HER2 concentration is the one used for the detection, in this case, it is the R3. The fitting analysis was performed on all the EIS Bode plots. This step is followed by the normalization of the data which is presented in Figure $3 \mathrm{~B}$ as absolute $\Delta \mathrm{R} 3 / \mathrm{R} 3$ (Whereas absolute $\Delta \mathrm{R} 3 / \mathrm{R} 3=$ IR3(HER2)-R3(antiHER2) I/R3(anti-HER2)) as a function of HER2 concentrations.

In order to test the sensitivity of the biosensor, the detection of HER2 was tested against the detection of two interfering proteins EGFR and HER3. Their respective detection yielded the following curves: HER2 $\left(\mathrm{Y}=(0.0062 \pm 0.0001) \times+(0.1066 \pm 0.0016) / \mathrm{R}^{2}=0.9992\right) ;$ EGFR $(\mathrm{Y}=(0.0016 \pm$ $\left.0.0006) \times+(0.0188 \pm 0.0086) / R^{2}=0.7214\right) ;$ and HER3 $\left(Y=(0.0035 \pm 0.0018) \times+(0.0225 \pm 0.0235) / R^{2}=\right.$ 0.5992).

(A)

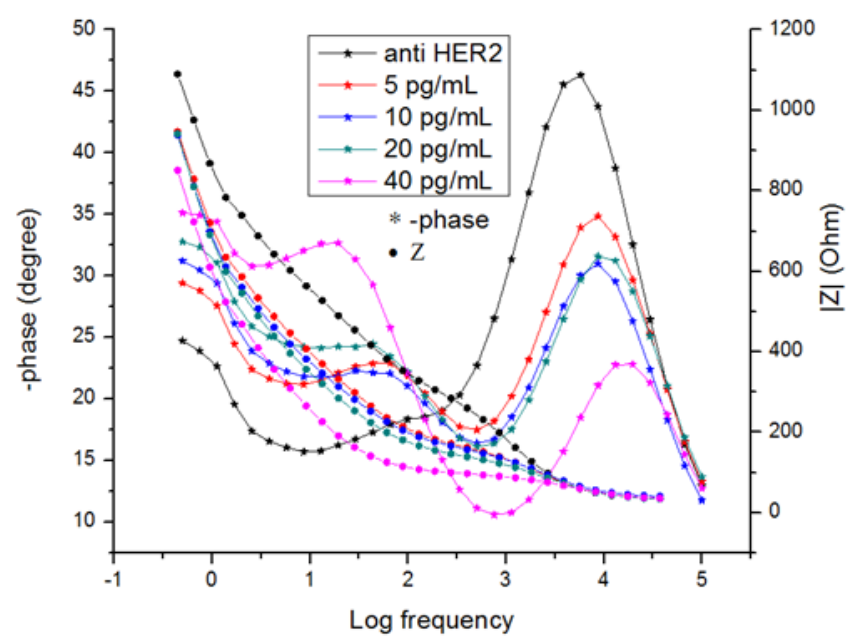

(B)

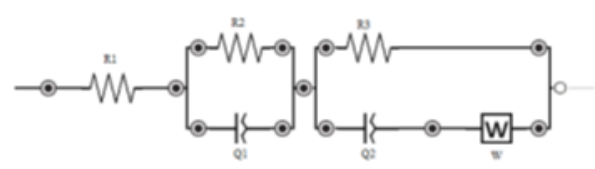

Figure 3. (A) Bode impedance plot (Log(frequency) vs -phase $\left(^{\circ}\right)$ vs $\mathrm{Z}(\mathrm{OHM})$ ) of $\mathrm{K} 3[\mathrm{Fe}(\mathrm{CN}) 6] / \mathrm{K} 4[\mathrm{Fe}(\mathrm{CN}) 6](5 \mathrm{mM})$ in PBS $(\mathrm{pH}=7.4)$ for various concentrations of HER2. (B) Equivalent circuit used to fit the resulting bode plots.

\subsection{Detection of HER2 in Real Saliva}

This is then followed by detecting HER2 in saliva through standard addition method as described in Section 2.4. The results, shown in Figure 4, of the detection of the concentration of the HER2 in saliva is seen in Figure 4, the linearity $\left(\mathrm{R}^{2}=0.9904\right)$ and the detection of the spiked HER2 concentration $(Y=(0.0118) \times+(0.1282))$, meaning the platform can be used in future work for detecting HER2 in saliva. 


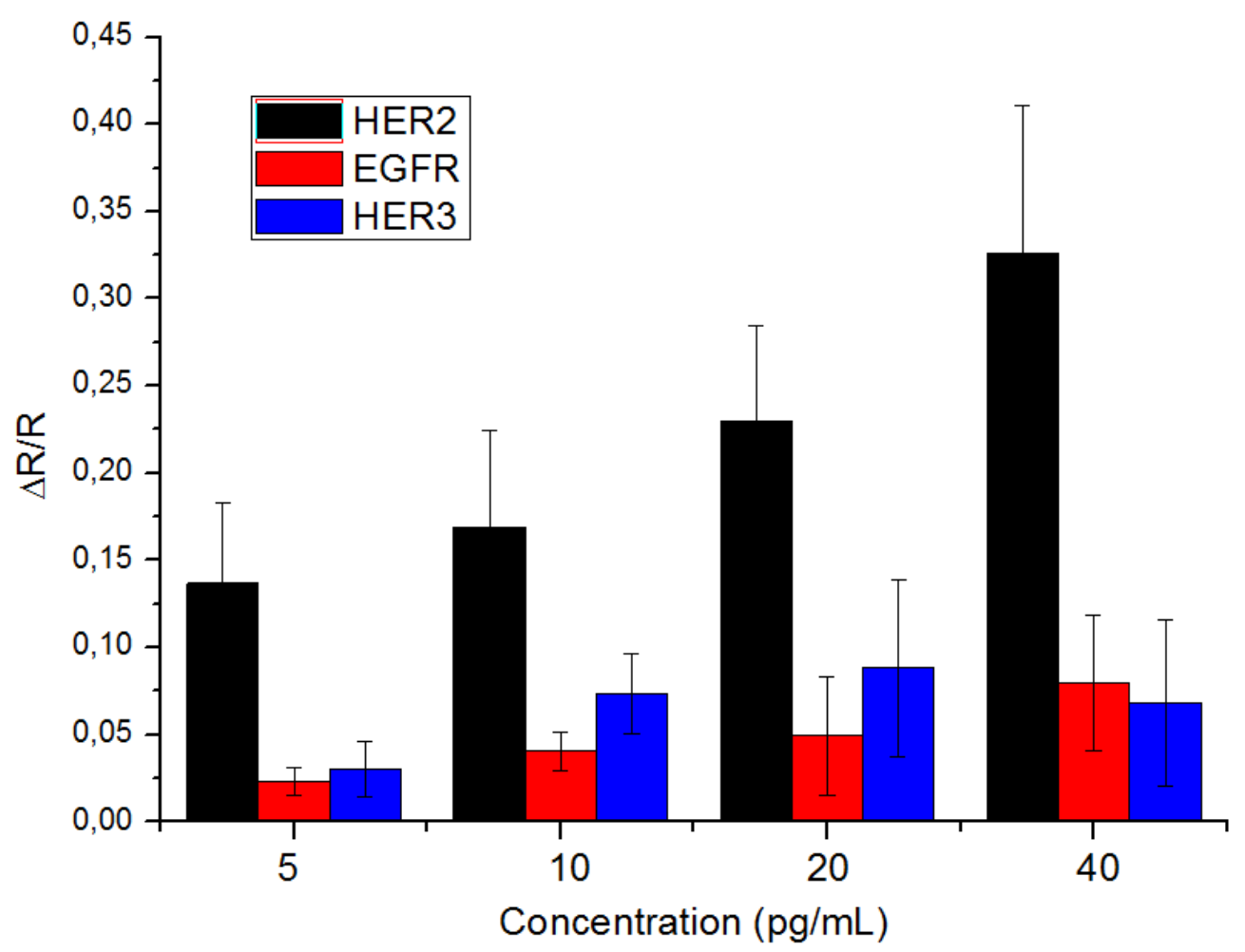

Figure 4. Detection histograms for the detection of HER2 ( $N=4$ times), EGFR ( $N=3$ times) and HER3 $(\mathrm{N}=4$ times) with the error being the calculated standard deviation for each detected protein.

\section{Discussion}

\subsection{Bode Plot and the Equivalent Circuit}

Due to the vast quantity of value a single measurement of EIS provides, several forms of representations were designed in order to show the value of any given measurement. In our case, bode plot proved to be more useful in terms of presentation of our measurement as it allowed us to deduce the equivalent circuit used to fit the data presented through the presentation of the number of peaks shown in the phase shift part of the graph [30]. From the Figure 3A, two distinct phase shift peaks are observed: the first starts at $\log$ freq of 5 and ends at $\log$ freq of 2.5; in this range, the phase angle peaks shift lower when the concentration of HER2 is increased. The other peak starts at log freq 2.5 and ends at $\log$ freq of 0 ; in this range the shift in the phase angle peaks increases with the increase concentration of HER2. Below the log freq of 0 there is a leveling off of the phase shifts indicating the end of the detection peaks. What this means is that the equivalent circuit of the system is made up of three charge resistances, two constant phase elements (CPE), and a Warburg element $\mathrm{W}$ as seen in Figure 3B.

In comparison, the impedance part of the plot shows a decrease with the increase concentration of HER2 in each of the steps presented in the graph, this decrease is most pronounced with the second step that corresponds with the second phase shift peak.

A similar circuit was described in the Nouira 2012 article [31] that highlights the presence of two electrochemical layers due to the presence of inter and intra nanoparticle regions. The observation of these two layers in these electrode confirms with the nature of the gold ink used in these screen printed electrode as them being porous electrodes as discussed in Barhoumi 2019's work on these electrodes [26,32].

The explanation of the circuits components are as follows: The charge resistances presented are as follow the solution resistance R1, the inter-gold particles resistance R2 which is presented in the high frequency peaks and the intra-gold particles $\mathrm{R} 3$ which is presented in the low frequency peaks. The CPE for the inter- gold particle is Q1 which is presented in the high frequency peaks, and for the 
intra-gold particle Q2 which is presented in the low frequency peaks, and finally the Warburg diffusion $W$.

\subsection{Detection of HER2 in PBS}

The biosensor's ability to detect HER2 is the responsiveness of the impedance response toward the increasing concentrations of HER2 in PBS. The concentration range chosen is perfect for the detection of salivary HER2 concentrations in healthy individuals and breast cancer patients alike $[17,33]$. The resulting fit of the bode plot showed excellent response to the concentrations of HER2, while repeated detection of HER2 have yielded a similarly repeatable curve of $Y=(0.0055 \pm 0.0003) \times$ $+(0.1127 \pm 0.0052) \backslash R^{2}=0.9909$ with high linearity, indicating high repeatability.

In order to test the biosensor's selectivity, additional tests were carried out using the same biofunctionalization steps as mentioned before only with the detection of biomarkers other than HER2. For that purpose, EGFR and HER3 were chosen. These biomarkers have a similar structure to HER2 $[7,34]$, while several studies have found the presence of EGFR in saliva $[17,33]$ it remains unclear whether HER3 is also present there. The result of the detection of the interferences shows that the biosensor is quite selective at this low concentration; the biosensor managed to detect HER2 with a sensitivity that is 4 times higher than that of EGFR $\left(Y=(0.0018 \pm 0.0005) \times+(0.0166 \pm 0.0049) \backslash R^{2}=\right.$ $0.8072)$ and twice higher than that of HER3 $\left(Y=(0.0020 \pm 0.0169) \times+(0.0530 \pm 0.0151) \backslash R^{2}=0.1302\right)$, thus proving the biosensor's selectivity toward HER2.

\subsection{Detection of HER2 in Saliva}

Standard addition method is one of the main analytical techniques used in determining an unknown concentration of analyte in complex matrices (biological fluids, soil samples, etc.). However, this detection only works if it's linearity continue for the entire working range of the analyte. [35]. The detection of HER2 through standard addition method have good linearity $\left(\mathrm{R}^{2}=\right.$ 0.9904 ) while also showing a detection of the concentration of around $10.86 \mathrm{pg} / \mathrm{mL}$ of HER2 in the real saliva. Thus proving that the biosensor can be used for the detection of HER2 in saliva.

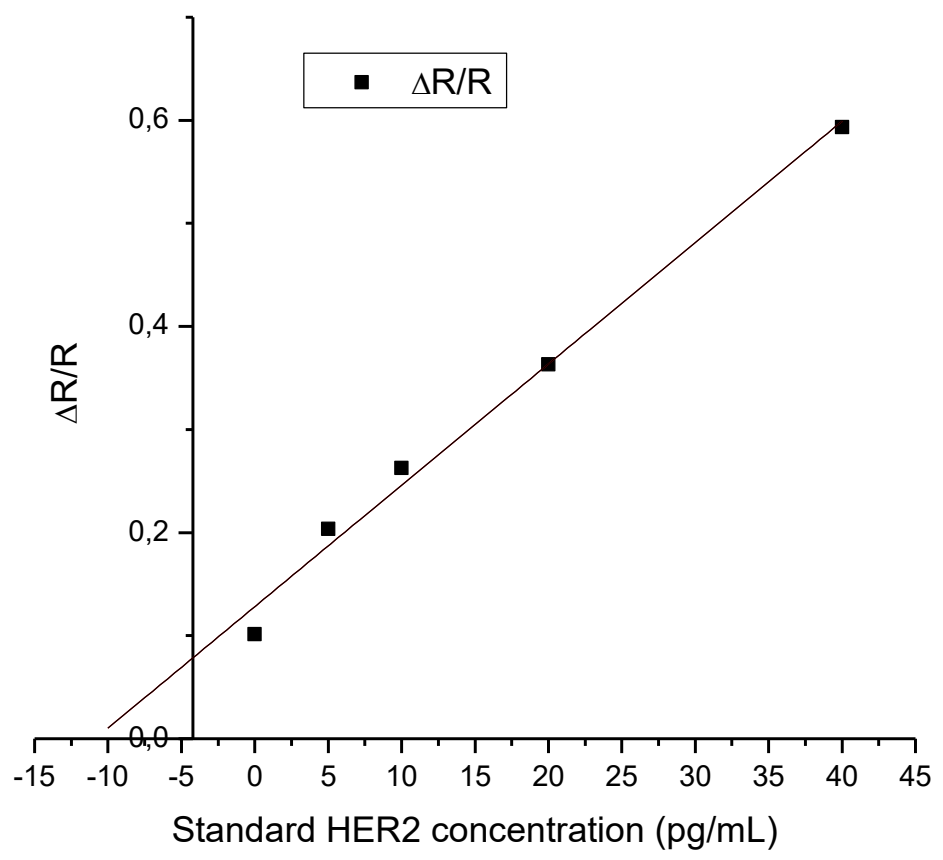

Figure 5. Result of the standard addition method for the detection of HER2 in real saliva $(\mathrm{N}=1)$. 


\section{Conclusions}

In conclusion, we would like to report the development of a label-free biosensor for the detection of HER2 in saliva. The biosensor uses integrated gold scree printed electrode as transducer, the biosensor is then biofunctionalized first by electrodeposition of ABA on the gold surface, followed by the immobilization of anti-HER2 antibody on the electrode surface. The biosensor uses EIS to detect the impedance of the biosensor in $\mathrm{Fe}^{2+} / \mathrm{Fe}^{3+}$. The result of the detection is a biosensor that is sensitive toward HER2 with high linearity that is 4 times more sensitive than the interference molecules EGFR and twice more sensitive than HER3. This is then followed by detecting HER2 in real saliva using standard addition method which proved to have great linearity $\left(R^{2}=0.9904\right)$ proving that this technique can be used to detect HER2 solution with real patients.

Author Contributions: I.A.N. contributed toward the realization of the experiments and the writing of the article. L.M. assisted with the realization of the experiment. A.E. and N.Z. contributed equally toward the design of the biosensor and provided their expertise in the field of electrochemistry and surface modification. W.H. and J.S. have contributed equally, they are the main coordinators and they provided their expertise in the experimental aspects of the research. All authors have read and agreed to the published version of the manuscript.

Funding and Acknowledgments: We would like to acknowledge the organization UK-Lebanon techhub for funding this research under their project Primispot.

Conflicts of Interest: We declare that there is no conflict of interest in this work.

\section{References}

1. Pilleron, S.; Sarfati, D.; Janssen-Heijnen, M.; Vignat, J.; Ferlay, J.; Bray, F.; Soerjomataram, I. Global cancer incidence in older adults, 2012 and 2035: A population-based study. Int. J. Cancer 2018, 58, 49-58.

2. Torre, L.A.; Bray, F.; Siegel, R.L.; Ferlay, J.; Lortet-tieulent, J.; Jemal, A. Global Cancer Statistics, 2012. CA Cancer J. Clin. 2015, 65, 87-108.

3. Ferlay, J.; Colombet, M.; Soerjomataram, I.; Mathers, C.; Parkin, D.M.; Piñeros, M.; Znaor, A.; Bray, F. Estimating the global cancer incidence and mortality in 2018: GLOBOCAN sources and methods. Int. J. Cancer 2018, 144, 1941-1953.

4. Duffy, M.J.; Walsh, S.; McDermott, E.W.; Crown, J. Biomarkers in Breast Cancer: Where Are We and Where Are We Going? 1st ed.; Elsevier Inc.: Amsterdam, The Netherlands, 2015; Volume 71, ISBN 9780128022566.

5. Nicolini, A.; Ferrari, P.; Duffy, M.J. Prognostic and predictive biomarkers in breast cancer: Past, present and future. Semin. Cancer Biol. 2018, 52, 56-73.

6. Rakha, E.A.; Green, A.R. Molecular classification of breast cancer: what the pathologist needs to know. Pathology 2017, 49, 111-119.

7. Omenn, G.S.; Guan, Y.; Menon, R. A new class of protein cancer biomarker candidates: Differentially expressed splice variants of ERBB2 (HER2/neu) and ERBB1 (EGFR) in breast cancer cell lines. J. Proteom. 2014, 107, 103-112.

8. Ferreira, P.M.P.; Pessoa, C. Molecular biology of human epidermal receptors, signaling pathways and targeted therapy against cancers: new evidences and old challenges. Braz. J. Pharm. Sci. 2017, 53, doi:10.1590/s2175-97902017000216076.

9. Greabu, M.; Battino, M.; Mohora, M.; Totan, A.; Didilescu, A.; Spinu, T.; Totan, C.; Miricescu, D.; Radulescu, R. Saliva--a diagnostic window to the body, both in health and in disease. J. Med. Life 2009, 2, 124-132.

10. Liu, J.; Duan, Y. Saliva: A potential media for disease diagnostics and monitoring. Oral Oncol. 2012, 48, 569577.

11. Abrao Nemeir, I.; Saab, J.; Hleihel, W.; Errachid, A.; Jafferzic-Renault, N.; Zine, N. The Advent of Salivary Breast Cancer Biomarker Detection Using Affinity Sensors. Sensors 2019, 19, 2373.

12. Malon, R.S.P.; Sadir, S.; Balakrishnan, M.; Córcoles, E.P. Saliva-Based Biosensors: Noninvasive Monitoring Tool for Clinical Diagnostics. Biomed Res. Int. 2014, 2014, 1-20.

13. Chojnowska, S.; Baran, T.; Wilińska, I.; Sienicka, P.; Cabaj-Wiater, I.; Knaś, M. Human saliva as a diagnostic material. Adv. Med. Sci. 2018, 63, 185-191. 
14. Yordanova, M.G.; Gerova, D.I.; Galunska, B.T. Saliva application in oral and systemic diseases. Scr. Sci. Medica 2018, 50, 13-18.

15. Streckfus, C.; Bigler, L.; Dellinger, T.; Dai, X.; Kingman, A.; Thigpen, J.T. The presence of soluble c-erbB-2 in saliva and serum among women with breast carcinoma: A preliminary study. Clin. Cancer Res. 2000, 6, 2363-2370.

16. Streckfus, C.F.; Arreola, D.; Edwards, C.; Bigler, L. Salivary protein profiles among her2/neu-receptorpositive and -negative breast cancer patients: Support for using salivary protein profiles for modeling breast cancer progression. J. Oncol. 2012, 2012, 413256.

17. Laidi, F.; Bouziane, A.; Lakhdar, A.; Khabouze, S.; Rhrab, B.; Zaoui, F. Salivary expression of soluble HER2 in breast cancer patients with positive and negative HER2 status. 2014, 7, 1285-1289.

18. Chiu, M.L.; Lawi, W.; Snyder, S.T.; Wong, P.K.; Liao, J.C.; Gau, V. Matrix Effects-A Challenge Toward Automation of Molecular Analysis. JALA-J. Assoc. Lab. Autom. 2010, 15, $233-242$.

19. Jayanthi, V.S.P.K.S.A.; Das, A.B.; Saxena, U. Recent advances in biosensor development for the detection of cancer biomarkers. Biosens. Bioelectron. 2017, 91, 15-23.

20. Barhoumi, L.; Baraket, A.; Bellagambi, F.G.; Karanasiou, G.S.; Ali, M. Ben; Fotiadis, D.I.; Bausells, J.; Zine, N.; Sigaud, M.; Errachid, A. A novel chronoamperometric immunosensor for rapid detection of TNF-A in human saliva. Sens. Actuators B Chem. 2018, 266, 477-484.

21. Baraket, A.; Lee, M.; Zine, N.; Sigaud, M.; Bausells, J.; Errachid, A. A fully integrated electrochemical biosensor platform fabrication process for cytokines detection. Biosens. Bioelectron. 2017, 93, 170-175.

22. Bahadir, E.B.; Sezgintürk, M.K. A review on impedimetric biosensors. Artif. Cells, Nanomed. Biotechnol. 2016, 44, 248-262.

23. Randviir, E.P.; Banks, C.E. Electrochemical impedance spectroscopy: An overview of bioanalytical applications. Anal. Methods 2013, 5, 1098-1115.

24. Karunakaran, C.; Rajkumar, R.; Bhargava, K. Introduction to Biosensors. Biosens. Bioelectron. 2015, 60, 1-68.

25. Taleat, Z.; Khoshroo, A.; Mazloum-Ardakani, M. Screen-printed electrodes for biosensing: A review (20082013). Microchim. Acta 2014, 181, 865-891.

26. Barhoumi, L.; Bellagambi, F.G.; Vivaldi, F.M.; Baraket, A.; Clément, Y.; Zine, N.; Ali, M. Ben; Elaissari, A.; Errachid, A. Ultrasensitive immunosensor array for TNF- $\alpha$ detection in artificial saliva using polymercoated magnetic microparticles onto screen-printed gold electrode. Sensors (Switzerland) 2019, 19, 692.

27. Radi, A.E.; Lates, V.; Marty, J.L. Mediatorless hydrogen peroxide biosensor based on horseradish peroxidase immobilized on 4-carboxyphenyl film electrografted on gold electrode. Electroanalysis 2008, 20, 2557-2562.

28. Campuzano, S.; Yánez-Sedeño, P.; Pingarrón, J.M. Electrochemical bioaffinity sensors for salivary biomarkers detection. TrAC-Trends Anal. Chem. 2017, 86, 14-24.

29. Abrao Nemeir, I.; Saab, J.; Hleihel, W.; Errachid, A.; Zine, N. Impedimetric label-free detection of salivary EGFR on screen printed electrode. Integr. Cancer Sci. Ther. 2019, 6, 1-3.

30. Huang, J.; Li, Z.; Liaw, B.Y.; Zhang, J. Graphical analysis of electrochemical impedance spectroscopy data in Bode and Nyquist representations. J. Power Sources 2016, 309, 82-98.

31. Nouira, W.; Maaref, A.; Vocanson, F.; Siadat, M.; Saulnier, J.; Lagarde, F.; Jaffrezic-Renault, N. Enhancement of Enzymatic IDE Biosensor Response Using Gold Nanoparticles. Example of the Detection of Urea. Electroanalysis 2012, 24, 1088-1094.

32. Lasia, A. Electrochemical Impedance Spectroscopy and Its Applications; 2014; Volume 9781461489, ISBN 9781461489337.

33. Streckfus, C.F.; Arreola, D.; Edwards, C.; Bigler, L. Salivary protein profiles among her2/neu-receptorpositive and -negative breast cancer patients: Support for using salivary protein profiles for modeling breast cancer progression. J. Oncol. 2012, 2012, 413256.

34. Sierke, S.L.; Cheng, K.; Kim, H.H.; Koland, J.G. Biochemical characterization of the protein tyrosine kinase homology domain of the ErbB3 (HER3) receptor protein. Biochem. J. 1997, 322, 757-763.

35. Bellagambi, F.G.; Baraket, A.; Longo, A.; Vatteroni, M.; Zine, N.; Bausells, J.; Fuoco, R.; Di Francesco, F.; Salvo, P.; Karanasiou, G.S.; et al. Electrochemical biosensor platform for TNF- $\alpha$ cytokines detection in both artificial and human saliva: Heart failure. Sens. Actuators B Chem. 2017, 251, 1026-1033.

Publisher's Note: MDPI stays neutral with regard to jurisdictional claims in published maps and institutional affiliations. 
(C) 2020 by the authors. Licensee MDPI, Basel, Switzerland. This article is an open access article distributed under the terms and conditions of the Creative Commons Attribution (CC BY) license (http://creativecommons.org/licenses/by/4.0/). 